carbon and loss-on-ignition values indicate accumulation of organic matter with increasing age of restoration. Age and position on transect had a marked effect on the bacterial community with respect to salt tolerance, but only small effects on numbers. The age of restoration had a marked effect on the numbers of fungal colony forming units, but only marginal effects on the distribution between the tolerance classes.

\title{
Distribution of Conidiobolus and Basidiobolus in managed ecosystems
}

\author{
I. J. Hopkins \\ Department of Chemistry and Biology, North Staffordshire Polytechnic ST4 2DE, \\ U.K.
}

An investigation of the occurrence of fungi of these genera in three contrasting ecosystems - an arable field, a pasture and a larch plantation - situated at Keele, Staffordshire was undertaken. The current study is a follow-up to an earlier investigation carried out in the same three habitats (Smith \& Callaghan 1987). Regular monthly soil sampling in all three habitats started in June 1986 and is still in progress. Improvements and refinements made to the original disclosure technique have substantially decreased the number of soil samples giving null returns of these fungi, and have resulted in an enhanced disclosure of certain small-spored species of Conidiobolus (e.g. C. lamprauges Drechsler) and of species producing capilliconidia.

The present investigation has reinforced the tentative conclusions drawn from the earlier study that the different habitats are to some extent characterised by different species. Basidiobolus ranarum Eidam and small-spored forms of Conidiobolus were found to be predominant in pasture soil, while Conidiobolus firmipilleus Drechsler and species forming capilliconidia were characteristic of the arable field. The most abundant forms in the larch litter were found to be Conidiobolus adiaeretus Drechsler, and a presumptive new Conidiobolus species, " $\alpha$ - new" (Waters \& Callaghan, in prep). Species occurrence was found to decline markedly with increasing depth except in the arable soil. Using the much fuller data for species occurrence obtained in the present study, attempts were made to correlate particular species peaks with the record of soil and air temperature, frosts and rainfall.

Further attempts were made to correlate changes in species occurrence and relative abundance with the timing of agricultural operations. These efforts have generally proved fruitless, although there are possible indications that the disclosure of certain species widespread in the larch litter (e.g. C.adiaeretus) may be influenced by the pattern of air frost over a period of days before sampling. In conclusion, this survey demonstrates the widespread occurrence in nature of many members of these relatively little studied genera of the Entomophthorales.

\section{References}

Smith, M. F. \& Callaghan, A. A. 1987. Quantitative survey of Conidiobolus and Basidiobolus in soils and litter. Transactions of the British Mycological Society 89, 179-185.

Waters, S. D. \& Callaghan, A. A. (in preparation). A new species of Conidiobolus forming actively discharged elongate repetitional conidia. 